\title{
Serotonin Affects Movement Gain Control in the Spinal Cord
}

\author{
Kunlin Wei, ${ }^{1}$ Joshua I. Glaser, ${ }^{2,3,4,5}$ Linna Deng, ${ }^{1}$ Christopher K. Thompson, ${ }^{5,6}$ Ian H. Stevenson, ${ }^{2,3,4,5}$ Qining Wang, ${ }^{1}$ \\ Thomas George Hornby, ${ }^{2,3,4,5,6}$ Charles J. Heckman, ${ }^{2,3,4,5}$ and Konrad P. Kording 2,3,4,5 \\ ${ }^{1}$ Department of Psychology, Peking University, Beijing, China 100871, Departments of ${ }^{2}$ Physical Medicine and Rehabilitation, ${ }^{3}$ Physiology, and ${ }^{4}$ Applied \\ Mathematics, Northwestern University, Chicago, Illinois 60611, ${ }^{5}$ Rehabilitation Institute of Chicago, Chicago, Illinois 60611, and ${ }^{6}$ Department of \\ Kinesiology and Nutrition, University of Illinois at Chicago, Chicago, Illinois 60607
}

A fundamental challenge for the nervous system is to encode signals spanning many orders of magnitude with neurons of limited bandwidth. To meet this challenge, perceptual systems use gain control. However, whether the motor system uses an analogous mechanism is essentially unknown. Neuromodulators, such as serotonin, are prime candidates for gain control signals during force production. Serotonergic neurons project diffusely to motor pools, and, therefore, force production by one muscle should change the gain of others. Here we present behavioral and pharmaceutical evidence that serotonin modulates the input- output gain of motoneurons in humans. By selectively changing the efficacy of serotonin with drugs, we systematically modulated the amplitude of spinal reflexes. More importantly, force production in different limbs interacts systematically, as predicted by a spinal gain control mechanism. Psychophysics and pharmacology suggest that the motor system adopts gain control mechanisms, and serotonin is a primary driver for their implementation in force production.

Key words: efficient control; gain control; neuromodulation; pharmacology; serotonin; spinal cord

\section{Introduction}

A central question in neuroscience is how neurons, with limited bandwidth, can encode signals that vary over multiple orders of magnitude. Gain control mechanisms, which are present in virtually all sensory systems, effectively solve this problem (Smirnakis et al., 1997; Zhao and Santos-Sacchi, 1999; Schwartz and Simoncelli, 2001; Chance et al., 2002). However, our motor system faces an analogous problem (Fullerton and Cattell, 1892; Carlton and Newell, 1993).

The forces we produce vary from fractions of a Newton, e.g., when we put a contact lens into our eye, to nearly $5000 \mathrm{~N}$, the world record in bench pressing. Because the forces we produce vary over multiple orders of magnitude but motor commands from the brain to spinal cord are transmitted by noisy neurons with limited bandwidth, a gain system would reduce noise in motor output. However, it is not currently known how, and even if, the spinal cord uses such a gain control mechanism to maintain accuracy over such a large range of forces.

If the motor system uses a gain control mechanism, how could it do so? All motor output from the trunk and limbs is generated

Received May 7, 2014; revised July 6, 2014; accepted Aug. 4, 2014.

Author contributions: K.W., J.I.G., L.D., C.K.T., I.H.S., Q.W., T.G.H., C.J.H., and K.P.K. designed research; K.W., J.I.G., L.D., C.K.T., I.H.S., Q.W., T.G.H., and C.J.H. performed research; K.W., J.I.G., L.D., C.K.T., I.H.S., T.G.H., C.J.H., and K.P.K. analyzed data; K.W., J.I.G., L.D., C.K.T., I.H.S., Q.W., T.G.H., C.J.H., and K.P.K. wrote the paper.

The study was supported by National Natural Science Foundation of China Grants 31371020, 31328010, 61005082, J1103602, and 61020106005, Beijing Nova Program Grant Z141101001814001, National High Technology Research and Development Program of China 863 Program Grant 2012AA011602, and National Institutes of Health Grants R01NS063399, P01NS044393, and R01NS034382. We thank Dr. Hongxiao Jia.

The authors declare no competing financial interests.

Correspondence should be addressed to Kunlin Wei, Department of Psychology, Peking University, Beijing, China 100871.E-mail: wei.kunlin@pku.edu.cn.

DOI:10.1523/JNEUROSCI.1855-14.2014

Copyright $\odot 2014$ the authors $\quad 0270-6474 / 14 / 3412690-11 \$ 15.00 / 0$ by motoneurons in the ventral horn of the spinal cord, whose axons connect directly to muscle fibers (Kernell, 2006). Previous physiological studies and modeling work suggested that neuromodulatory gain control is necessary for force production, because even the "maximal effort" (maximum current in motoneurons) would scarcely produce $40 \%$ of maximal force without neuromodulatory input (Cushing et al., 2005; Heckman et al., 2008). A potent neuromodulatory drive to these motoneurons comes from the brainstem via axons projecting monosynaptically onto motoneurons and releasing either serotonin (5-HT) or norepinephrine (NE; Bowker et al., 1982; Holstege and Kuypers, 1987). Both of these neuromodulators facilitate voltage-sensitive changes on spinal motoneurons, resulting in greatly increased input-output excitability, i.e., gain (Heckman et al., 2003; Hultborn et al., 2004).

5 -HT is more likely to be involved in gain control, because 5-HT projection to the spinal cord increases its activity with increasing motor output (Jacobs et al., 2002), whereas the NE system covaries with state of arousal (Aston-Jones et al., 2001). This evidence leads to the hypothesis that 5-HT in the spinal cord plays a significant role in a motor output gain control system (Jacobs et al., 2002).

Here, we first aim to establish that 5-HT alters gain in the spinal cord. If this is true, drugs that augment/suppress 5-HT should also enhance/inhibit spinal reflex, which is heavily influenced by excitability of spinal motoneurons. Second, we aim to show that this spinal gain mechanism is present during force production. If this is true, because of the diffuse projection of 5-HT into the spinal cord, intense contraction of one muscle group (which requires high gain) should degrade the precision at which a subsequent low-force motor task can be achieved with other muscles. Third, we aim to show that 5 -HT affects force 
a

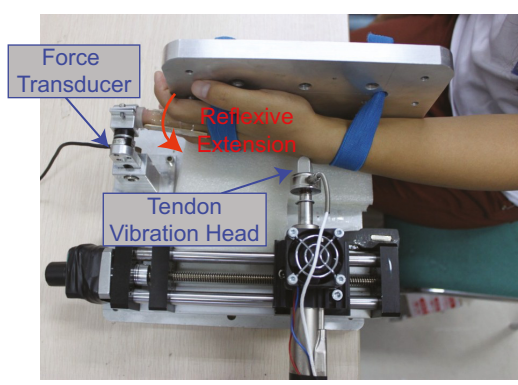

b

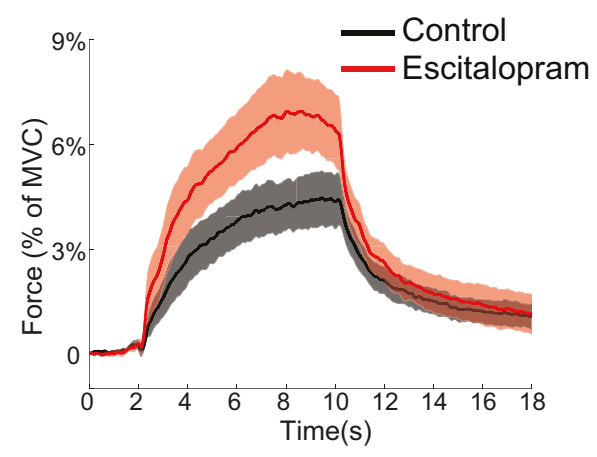

nary trials for the experimenter to adjust the vibration head and for subjects to get acquainted with experimental procedures.

Experiment 2 measured the tendon reflex response as a function of drug intake in the lower extremity of individuals with reduced descending drive. Seven subjects with chronic $(>1$ year) spinal cord injury participated in two sessions separated by at least $5 \mathrm{~d}$ : (1) one session with $20 \mathrm{mg}$ of escitalopram intake; and (2) one with $8 \mathrm{mg}$ of cyproheptadine intake (administrated double-blinded). The order of the two sessions was randomized among subjects. In each session, data collection occurred before drug intake and $5 \mathrm{~h}$ after drug intake. Subjects were seated in the adjustable height chair of the testing apparatus (System 3; Biodex Medical Systems) with the hips flexed to $45^{\circ}$ and the knee positioned at either $90^{\circ}$. Knee extension (KE) torques and surface electromyographic (EMG) data were collected on all subjects on the more impaired limb as determined during clinical evaluation, with the

production through this spinal gain mechanism. If this is true, drugs that augment 5-HT should increase variability in precision tasks, whereas those that block its effects should decrease variability. Our experiments test these predictions in human subjects.

\section{Materials and Methods}

Experiment details. Experiment 1 measured tendon vibration reflexes elicited on the left wrist as a function of drug intake. Subjects $(n=9)$ participated in two sessions on 2 consecutive days; (1) one session with escitalopram intake; and (2) one with placebo (administrated doubleblinded). The order of the two sessions was randomized among subjects, with four subjects taking the drug in the first session and the other five taking the drug in the second session. The data collection started $5 \mathrm{~h}$ after drug intake when the serum level of escitalopram reached its peak. Sitting before a desk, subjects put the tip of their left middle fingers snugly into a metal ring, which was firmly mounted on the desk top (Fig. 1a). Each subject's middle finger was also splinted, by two wooden sticks on the lateral sides that were bundled by medical tape, to minimize the movements between phalangeal joints. The metal ring was fixed onto a force transducer (resolution, $0.014 \mathrm{~N}$; model Nano 17; ATI) so the reflexive force could be measured. The left arm straightened out and was supported on the table; the left palm faced rightward and rested against a metal surface. A 2-cm-wide, concave-shaped metal head pointed to and pressed against the left wrist to apply a vibrating stimulus. The head was screwed on one side of a force transducer (resolution, $0.28 \mathrm{~N}$; model Sensotec 31; Honeywell); the other side of the force transducer was screwed onto a linear motor (model PS01-23x80; LinMot). The data collection was organized as trials. For each trial, the initial position of the vibration head was adjusted so that the contact force between the head and the wrist, measured from the attached force transducer, was above 4 N. Subjects sat idle with eyes closed after hearing a computer speakergenerated beep. After a random period of 2-4 s, the linear motor started to apply $100 \mathrm{~Hz}$ sinusoidal movements vertically to the wrist with a peak-to-peak displacement of $0.6 \mathrm{~mm}$. The vibration lasted $8 \mathrm{~s}$, and the data collection continued for another $10 \mathrm{~s}$, until a beep signaled the end of the trial. Subjects were instructed to remain relaxed through each trial. The contact force between the vibration head and the wrist was constantly monitored throughout the experiment; if it dropped below $4 \mathrm{~N}$, the experimenter would adjust the initial position of the head manually before the next trial started. This ensured that all trials had the same initial contact force. The tendon vibration elicited a wrist extension, which in turn generated a pushing force onto the force transducer attached to the finger ring. We also measured the maximum voluntary contraction (MVC) of this finger extension before the vibration trials started. A total of 32 vibration trials were collected: eight trials per block with an interblock rest period of $3 \mathrm{~min}$. The first 12 trials were prelimi- same limb being tested during all sessions. The distal shank of the tested limb was secured to the dynamometer arm, which was coupled to a 6 degree of freedom load cell (theta; resolution, $0.025 \mathrm{Nm}$; ATI) used to assess KE torques. Surface EMG was recorded using active bipolar electrodes (Delsys) applied over the vastus lateralis (VL), vastus medialis (VM), and rectus femoris (RF). A 2-cm-wide convex rubber head was aligned to the patellar tendon. The head was affixed to a load cell (resolution, $0.15 \mathrm{~N}$; Omegadyne), which in turn was attached in series to the end of the neodymium slider of a linear motor (model P01$23 \times 160 \mathrm{H}-\mathrm{HP}$; LinMot). A position control strategy was used to alter forces delivered to the tendon; forces were varied by moving the initial position of the tapper relative to the tendon using a constant $30 \mathrm{~mm}$ stroke. Reflex threshold was identified as the minimum distance necessary to elicit EMG responses of at least one KE muscle. The force applied to the tendon was controlled by delivering taps at 11 different positions ranging from the starting position of reflex threshold (i.e., $0 \mathrm{~mm}$ ) and moving progressively closer to the tendon in randomly ordered $1 \mathrm{~mm}$ steps such that responses from 11 different starting positions were assessed. The minimum rest between tendon reflexes was $20 \mathrm{~s}$, and responses were elicited two to three times at each position in a blocked manner.

Experiments 3-5 and the control experiments used similar paradigms. Subjects were seated in front of a table. In separate sessions of Experiment 3 , they either produced a leftward force against a strain gauge (resolution, $0.056 \mathrm{~N}$; model Gamma; ATI) fixed on the table top with the right index finger or the right palm or lifted the tips of their feet up against a wooden fixture placed directly above the feet. When using the finger or the palm to push against the strain gauge, the subject's right forearm was inserted into a customizable polyvinyl chloride tube to prevent arm movement. When using two feet, two Nintendo Wii Fit force sensors (resolution, $0.39 \mathrm{~N}$ ) were placed above the two feet to measure the upward force. The target force levels (low, medium, and high) were set at 5, 35, and 65\%, respectively, of MVC for each of the three effectors (finger, palm, and leg) and were represented as a horizontal line on a computer screen placed in front of the subject. The power forces produced by these effectors were measured and displayed in real time as a moving cursor on the screen. The vertical displacement of the cursor was controlled by the force magnitude, and the horizontal displacement was driven by elapsed time. Each trial was randomly assigned a target force level, and the subject was required to ramp up the power force in the first second and to maintain it precisely at the target level. At $3 \mathrm{~s}$, a monophonic beep signaled the subject to press the left index finger downward on a strain gauge (resolution, $0.0035 \mathrm{~N}$; model Nano 17; ATI). This precision force needed to reach its target level ( $5 \% \mathrm{MVC}$, constant across all sessions) in $1 \mathrm{~s}$ and be maintained there until the 14th second when the trial end. The target level was marked as a red horizontal line on the computer screen. At $5.8 \mathrm{~s}$, 
a stereophonic tone signaled subjects to stop producing the power force. As the power force ceased at approximately the 6th second, the two forces were simultaneously applied for $\sim 2 \mathrm{~s}$. The subjects were instructed to disengage the power force by gently releasing the right index finger, the right palm, or two feet from the force sensors, minimizing its impact on the precision force production. To minimize mechanical coupling between the two forces, the power force was applied in the lateral (finger and palm) or upward (leg) direction, whereas the precision force was always applied downward. A monetary reward, in reverse proportion to the variance of the precision force, was shown on the top-left corner of the computer screen after each trial to encourage good performance.

Experiment 3 consisted of three separate sessions, one for each effector used to produce the power force. The sequence of sessions was randomized among seven subjects. For each session, we first calibrated the force sensors by asking subjects to place their left index finger on the strain gauge and their feet below the foot fixture. The forces collected were averaged for zeroing the readings of the sensors. Then, MVCs of effectors for the power force and for the precision force were collected twice with a mandatory $90 \mathrm{~s}$ rest between. The larger force among the two readings was taken as the MVC for the session. Before formal data collection, subjects practiced the task on the apparatus for six trials. Trials in one session were further partitioned into four blocks of 12 trials each with a $90 \mathrm{~s}$ rest between sessions. The resting time between trials was $10 \mathrm{~s}$. MVCs were measured again in the middle (immediately after the second session) and after the experiment (immediately after the fourth session).

Experiments 4 and 5 only used the right palm as the effector for the power force. Both experiments had different sets of eight subjects, and they were measured in two sessions on 2 successive days: (1) one with drug intake; and (2) the other with placebo intake (administered doubleblind). The two sessions were performed at the same time during the day for each subject. To minimize the learning effect, the order of the two sessions was randomized among subjects, with four subjects taking the drug on the first day and the other four taking it on the second day. In Experiment 4, data collection started $2 \mathrm{~h}$ after oral intake of cyproheptadine or the placebo, because serum level of cyproheptadine usually reaches its peak level in $2 \mathrm{~h}$. For the same reason, data collection for Experiment 5 was started $5 \mathrm{~h}$ after paroxetine intake. Both experiments used the same protocol as Experiment 3 with minor changes: subjects practiced for 30 trials before formal data collection to minimize the learning effect, and the number of trials within each block increased to 15 .

Control Experiments A and B had the identical protocol as Experiment 3 with some modifications. Only the palm was used to produce the power force. Control Experiment A reversed the order of the precision force and the power force: the precision force reached its target level in the first second and maintained there until the trial end at the 16th second. The power force was ramped up between the second and the third seconds and then dropped at the eighth second. The duration of the power force thus remained $5 \mathrm{~s}$ to facilitate comparisons between experiments. Compared with Experiment 3, Control Experiment B switched the roles of two hands: the dominant hand produced the precision force, and the nondominant hand produced the power force. Eight subjects participated in Control Experiment A, and a different set of seven subjects participated in Control Experiment B. The same seven subjects then performed Control Experiment $\mathrm{C}$ in which only the precision force was produced with the right finger for 18 trials.

In Experiment 1, MVCs for the left middle finger extension were $7.65 \pm 0.54$ and $7.40 \pm 0.65 \mathrm{~N}$ with escitalopram intake and without, respectively. In Experiment 3, MVCs were $3.98 \pm 1.23$ (mean \pm SD), $10.86 \pm 3.30,23.35 \pm 6.87$, and $48.66 \pm 13.63 \mathrm{~N}$ for the left finger, right finger, right palm, and the average of both legs, respectively. In Experiment 4, MVCs for the left finger were $4.50 \pm 0.88$ and $4.61 \pm 0.67 \mathrm{~N}$ with cyproheptadine intake and without, respectively. MVCs for the right palm were $31.19 \pm 5.50$ and $31.37 \pm 6.89 \mathrm{~N}$, respectively. In Experiment 5 , MVCs for the left finger were $4.78 \pm 0.63$ and $5.06 \pm 0.29 \mathrm{~N}$ with paroxetine intake and without, respectively. MVCs for the right palm were $41.12 \pm 6.73$ and $40.71 \pm 5.95 \mathrm{~N}$, respectively. All subjects participated in experiments after providing informed consent. Subjects with motor incomplete spinal cord injury in Experiment 2 were recruited from a nonpublic registry housed within the Rehabilitation Institute of Chicago. They were seven males with chronic ( $>1$ year) spinal lesions above the T10 neurological level. Injuries were incomplete, and all subjects demonstrated residual volitional KE strength in the tested limb as determined by the lower extremity motor score (Marino and Graves, 2004). Exclusion criteria included medical history of multiple CNS lesions, known reaction to study medications, and diagnosis of lower limb peripheral nerve injury or orthopedic injury that may limit maximal effort during KE contractions.

All procedures of Experiment 1 and Experiments 3-5 and control experiments were approved by the ethics committee of Peking University. All procedures of Experiments 2 and 3 were approved by the Institutional Review Board of Northwestern University. All experiments were performed in accordance with the Declaration of Helsinki.

Data analysis. For Experiment 1, the force elicited by the tendon vibration reflex was measured at $1000 \mathrm{~Hz}$. It was low-pass filtered at $5 \mathrm{~Hz}$ by a fifth-order Butterworth filter to remove the measurement noise and the vibration force transmitted from the wrist to the finger. The force data were then normalized by dividing by individuals' MVCs. To derive the average force curve, all trials were aligned at $2 \mathrm{~s}$ before the vibration (time 0 ). We were particularly interested in the speed of force development immediately after the initiation of the vibration. This force rate was quantified by fitting a linear slope to the force data between the second and fourth seconds. We also calculated the average force level achieved during the last $2 \mathrm{~s}$ of the vibration. Thirteen trials (3.6\% of total trials) were excluded from analyses because visual inspection revealed that subjects occasionally failed to follow the instruction, e.g., moving their fingers before the vibration.

For Experiment 2, the force delivered to the tendon and resulting torque and EMG responses were collected at $1000 \mathrm{~Hz}$. The force signal was filtered at $220 \mathrm{~Hz}$, the torque signal was low-pass filtered at $200 \mathrm{~Hz}$, and the EMG signals were bandpass filtered at $20-450 \mathrm{~Hz}$ before digitization. The peak tap force for each tap was found offline and defined as tap onset. The peak-to-peak amplitude of the tendon reflex was calculated for each muscle. To assess the gain of the reflex response, the reflex amplitudes at the 11 different tap conditions were plotted against tap force to construct a reflex response curve for each muscle. The gain of the tendon reflex was calculated as the linear slope of this reflex response curves for each muscle. One subject was excluded from the analysis in the cyproheptadine group because tendon reflexes were unable to be elicited both before and after medication. To facilitate cross-muscle comparison, we calculated the percentage change in both the amplitude and gain measurements for each muscle. They were calculated as the difference between the post-medication and pre-medication values divided by premedication values and expressed as a percentage. We tested whether the percentage change across muscles in reflex amplitude and gain are different from 0 , after either escitalopram or cyproheptadine administration. Given the sample size and a lack of data normality, Wilcoxon's signed-rank tests were used.

For Experiments 3-5 and Control Experiments A and B, the precision force was sampled at $200 \mathrm{~Hz}$ and the power force at $75 \mathrm{~Hz}$. The precision force was bandpass filtered between 2 and $30 \mathrm{~Hz}$ with a fifth-order Butterworth filter to remove slow transients and high-frequency, nonphysiological measurement noise. The resulting data were aligned to the time when the power force dropped to $50 \%$ of its target value. The SD was calculated over each second before and after this time. SDs obtained were then normalized by dividing by the target force magnitude (Schmidt et al., 1979; Newell and Carlton, 1988). Thus, force variance is essentially quantified by the coefficient of variation $(\mathrm{CV})$. This measure takes individual differences in the target force into account and facilitates the comparison across participants. The mean over trials of each condition was reported. The criteria for eliminating trials included the following: (1) failure to start the precision force or to stop the power force within a $2 \mathrm{~s}$ window of the required time; (2) failure to stabilize the precision force during the $1 \mathrm{~s}$ before the power force stopped; and (3) failure to keep the precision force within 4 SDs of the target force during the stationary part of the trial after the power force ceased. In total, $6.7,4.0$, and $3.5 \%$ of all trials were eliminated for additional analysis in the three experiments, respectively. The number of eliminated trials did not differ between three 
force conditions (one-way ANOVA with $p=0.94,0.50$, and 0.76 , respectively). Data were examined for normality before being submitted to parametrical statistical analyses. The comparisons between force conditions (and between effectors) were conducted by one-sided paired $t$ tests. The comparisons between drug and force conditions were conducted by two-way ( 2 drug conditions $\times 3$ force levels) repeated-measures ANOVAs. The comparisons between force conditions in terms of variance changes induced by drugs were conducted by one-sided paired $t$ tests.

\section{Results}

\section{Evidence from spinal reflexes}

To establish that 5-HT serves as a gain control signal on the spinal motoneuron in humans, we examined the reflex response of tendon vibration among healthy subjects under the influence of escitalopram (Experiment 1; Fig. 1a). Previous studies in animal preparations have established that 5-HT strongly potentiates spinal reflexes (Carp and Rymer, 1986; Lee and Heckman, 2000). Escitalopram is a selective 5-HT reuptake inhibitor that can amplify the levels of 5-HT. The tendon vibration primarily activates muscle spindle Ia afferents that monosynaptically project to spinal motoneurons and is thus a spinally mediated response (Matthews, 1972). Thus, it allows us to experimentally ask how 5-HT affects spinal excitability.

To establish the methods we are using, we find that subjects produced similar MVCs with and without the drug $(p=0.57$, paired $t$ test). With tendon vibration, subjects immediately developed an extension force until reaching its plateau (Fig. 1b). The force dropped gradually after the vibration was turned off. All subjects reported after the experiment that their hand appeared to be moved passively, indicating that the tendon vibration reflex had been successfully elicited (Naito et al., 2002).

We found that the rate of force development, driven by constant tendon vibration stimuli, was significantly higher with escitalopram intake than without $(p<0.01$, paired $t$ test). The average force rates were $1.35 \pm 0.39$ and $2.2 \pm 0.45 \%$ of MVC per second for the placebo and escitalopram conditions, respectively. The achieved force was also significantly higher as evaluated during the last $2 \mathrm{~s}$ of vibration ( $p<0.05$, paired $t$ test). The average forces were $4.4 \pm 0.76$ and $6.8 \pm 1.1 \%$ of MVC for the placebo and escitalopram conditions, respectively. Hence, the enhanced efficacy of 5-HT leads to larger responses of tendon vibration reflex and thus supports 5-HT altering the gain of spinal motoneurons.

To further establish the role of 5-HT and also show that descending drive is unlikely to contribute to these findings, we examined magnitude and gain of tendon tap reflex responses in individuals with reduced descending drive attributable to partial spinal injury (Experiment 2). The tap evokes a brief activation of the same muscle spindle Ia afferents stimulated by tendon vibration (Matthews, 1972). Whereas the long duration of the tendon vibration reflex could potentially evoke non-spinal pathways, the tendon reflex elicited by brief taps is unequivocally spinal, mediated by monosynaptic EPSPs in motoneurons (Matthews, 1972). We systematically varied the amplitude of the tap, allowing direct measurement of reflex gain across a wide input-output range (Fig. 2).

Acute administration of serotonergic agents did indeed modify both the amplitude and gain of the patellar tendon reflex response across the quadriceps muscles (Fig. 2). After administration of escitalopram, the amplitude of the tendon reflex is increased by a median of $36.3 \%$ (range: $18.0-129.4$ ) across all muscles; likewise, the gain of the tendon reflex is increased by $196.1 \%$ (45.3-656.0), with both metrics being significantly greater than $0(p<0.0001$ and $p=$ 0.0001 , respectively; $n=7)$. The opposite effects were observed in response to a 5-HT antagonist. Here, acute administration of cyproheptadine decreased the amplitude of the tendon reflex by
$59.6 \%(35.3-73.1)$ and decreased the gain of the tendon reflex by $70.1 \%(51.5-80.3 ; p=0.0002$ and $p=0.0005$, respectively; $n=$ $6)$. These data support modification of the gain of tendon tap reflex by the serotonergic system.

\section{Why would a gain control mechanism be useful?}

Would the use of two distinct transmission channels (descending drives and gain) allow for more efficient transmission? We want to highlight the advantage of gain control with a simple example. Let us say we have a transmission system (e.g., motoneurons to muscles) that can only transmit a maximal number of spikes during a relevant interval and that we can only produce forces that are proportional to the number of spikes. Let us say that our system can produce a maximum of 10 spikes, and there is one situation (A) in which we need forces between 0 and $1 \mathrm{~N}$ and another situation (B) in which we need forces between 0 and $10 \mathrm{~N}$ (Fig. 3a). In this example, if we have a system with a fixed gain, then we must use a gain of $1 \mathrm{~N} /$ spike because a lower gain would not support the maximal forces needed in Situation B. However, this means that, in Situation A, we will only be able to produce integer-valued forces, resulting in errors up to $0.5 \mathrm{~N}$. Without a gain factor, the need to be able to produce high forces necessitates high errors for small forces.

If we can instead use a context-dependent gain, then we could use a gain of $0.1 \mathrm{~N} /$ spike in Situation A and $1 \mathrm{~N} /$ spike in Situation B. In this case, we will make maximal errors in Situation A of 0.05 $\mathrm{N}$, leading to a significant reduction in noise while leaving the errors in Situation B unaffected. Thus, in a limited bandwidth transmission system such as the brain, a gain system can result in more efficient transmission.

\section{Model of force production with gain control}

To be able to experimentally test the results of gain control, we need to specify a model of how gain control affects force output. With a gain control mechanism, muscular force is jointly determined by descending neural commands and gain signals. In a simple model, a desired force $F$ is the result of the descending drive multiplied by a spinal output gain: $F=$ gain $\times$ drive. The drive needed to produce a given force thus depends on the output gain: a high descending drive at low gain can yield the same average force as a low descending drive at high gain (Fig. 3b). However, these situations may differ in terms of their variability. Uncontrolled variability (noise) in force production is not solely defined by the descending drive but can be influenced by gain control at the motor output stage in the spinal cord.

Assuming that the pool of neurons transmitting the drive has Poisson-like behavior, as is typical for neurons in both sensory and motor systems (Churchland et al., 2010), the variance in the drive is proportional to the mean drive: $\sigma_{\text {drive }}^{2}=\alpha \mu_{\text {drive }}$, where $\alpha$ characterizes the Poisson-like behavior (the Fano factor; Teich et al., 1997). For a fixed gain, the SD in the force output relative to the target force $F$ will depend on the gain:

$$
\sigma_{F}=\text { gain } \sqrt{\alpha=\mu_{\text {drive }}} .
$$

In our model, we find that the lower the gain, the lower the noise. Thus, we can quantify gain control during force production by examining variability because force variability depends on the gain.

\section{Behavioral evidence from across-effector tasks}

Our behavioral experiments were designed based on evidence of a diffuse serotonergic system. Studies in animal preparations have established that the brainstem-spinal neuromodulatory sys- 
a
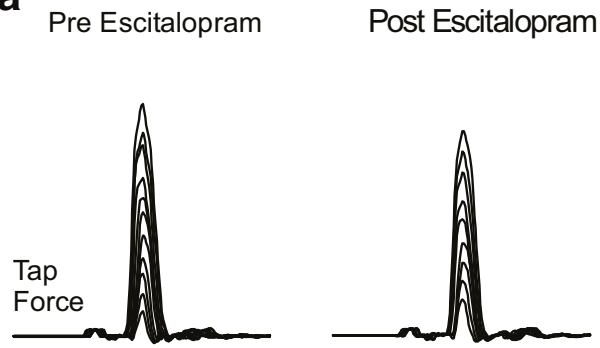

RF

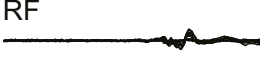

VL
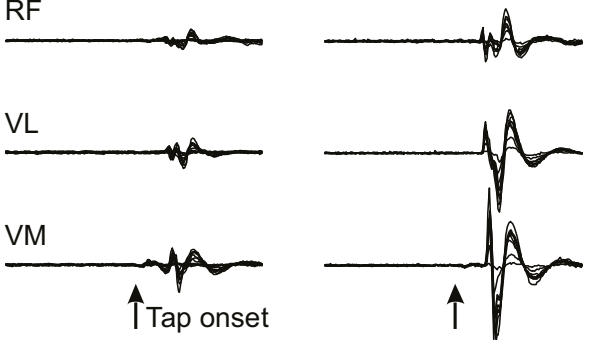

C

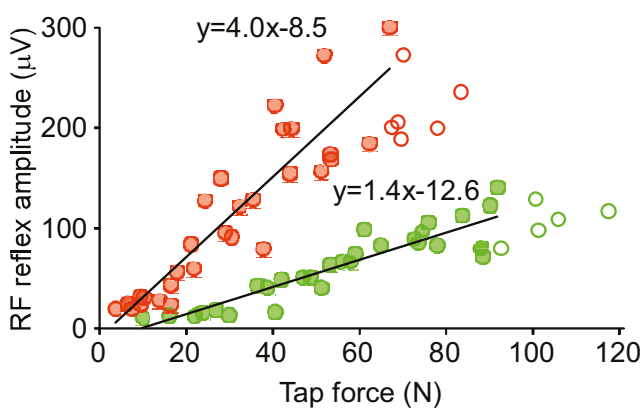

$\mathbf{e}$

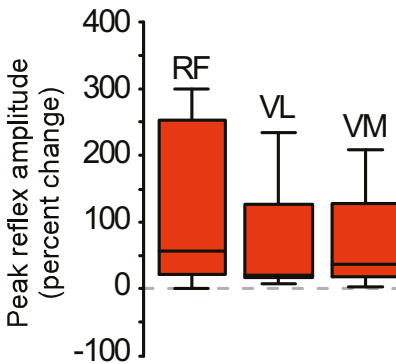

b

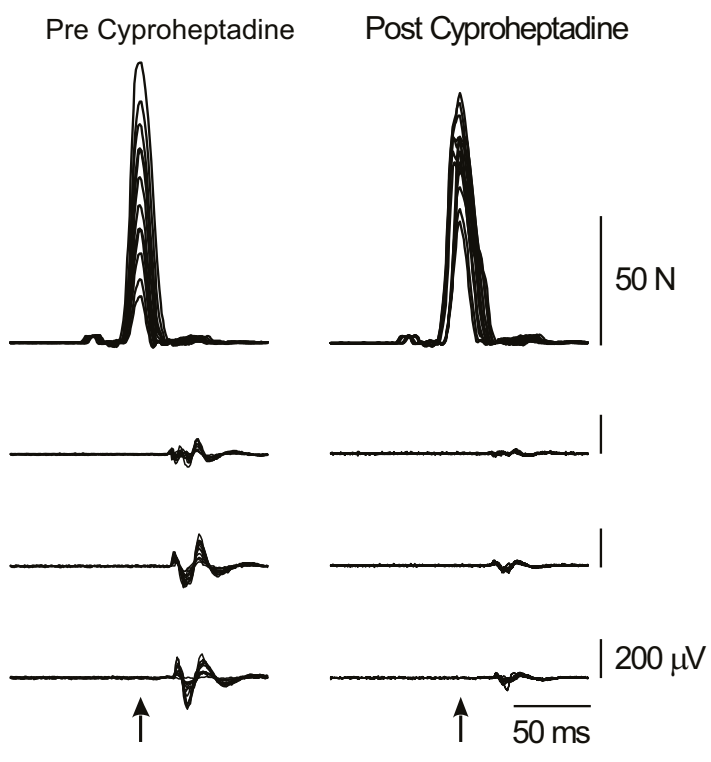

d
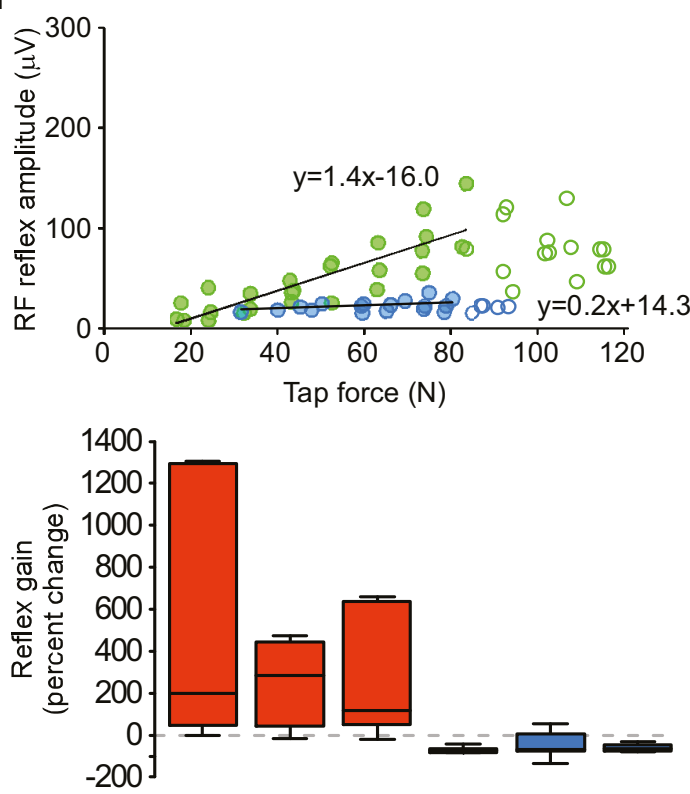

Figure 2. Results of Experiment 2 with individuals with chronic spinal cord injury (SCI). $\boldsymbol{a}$, Tap forces and elicited EMG responses for quadriceps tendon reflexes in a typical subject with and without escitalopram intake. Muscles include VL, VM, and RF. $\boldsymbol{b}$, Similar exemplary trials in the same subject as in $\boldsymbol{a}$ with administration of cyproheptadine. $\boldsymbol{c}$, The peak-to-peak amplitude of the reflex response for RF is plotted against tap force, and the reflex gain is calculated as the slope of the linear regression of the linear portion of the reflex curve (filled circles). Data are from the same typical subject before and after escitalopram intake. $\boldsymbol{d}$, Similar plot as in c from the same subject before and after cyproheptadine intake. $\boldsymbol{e}$, Across all subjects, escitalopram intake produces an increase in amplitude of the tendon reflex, producing a median $56 \%$ increase in the RF, $20 \%$ increase in the VL, and $36 \%$ increase in the VM, whereas after cyproheptadine intake, the amplitude of the tendon reflex is decreased by 61,48 , and $59 \%$ in the RF, VL, and VM, respectively. $f$, The gain of the reflex was likewise modified by serotonergic agents. After escitalopram intake, the gain of the response was increased by 196, 283, and 116\%, and after cyproheptadine intake, the gain of the response was decreased by 75,70 , and $65 \%$ in the RF, VL, and VM, respectively.

tem is highly diffuse in its projection from the brainstem to the spinal cord (Bowker et al., 1982; Holstege and Kuypers, 1987; Heckman et al., 2008). Similarly, studies in humans have suggested that the excitability of the corticospinal pathway can be enhanced with large concurrent force production in a separate effector (Devanne et al., 1997). This suggests that gain control mechanisms and neuromodulatory signals in particular affect the overall excitability of motor output throughout a limb or even across the whole body. Furthermore, gain control also changes relatively slowly and affects many neurons at the same time (Schwartz and Simoncelli, 2001); it is diffuse in space and time. Therefore, if there is a spinal gain control mechanism, we would expect force production in one effector to alter the gain of the corticospinal pathway and thus alter force production in a separate effector. Furthermore, this gain effect should last for a short period of time after the original effector stops its force production.

In Experiments 3-5, we used a psychophysical task requiring two successive isometric muscular contractions using separate effectors; we call the first the power force and the second the precision force. In Experiment 3, the power force is produced in three separate conditions by the right index finger, the right palm, or the two feet, and the intensity of the force is varied (Fig. $4 a$ ). In Experiments 4 and 5, the power force is produced by the right palm only, but subjects are required to take the drugs that selec- 
a

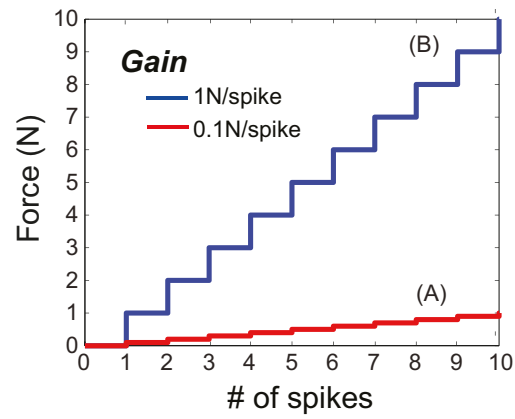

b

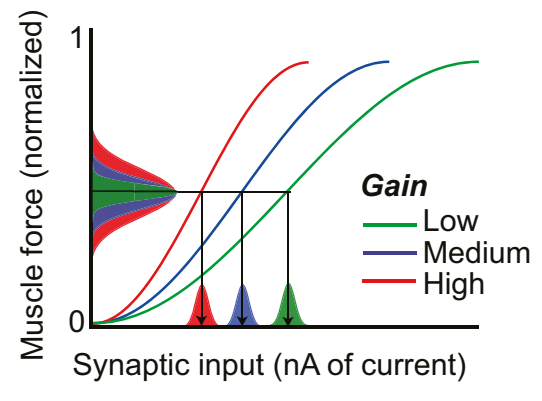

Figure 3. Model of gain control. $\boldsymbol{a}$, A hypothetical scenario with context-dependent gain control. To encode both 10 and $1 \mathrm{~N}$ target forces with only 10 spikes (B), the best resolution for the $1 \mathrm{~N}$ force is $0.5 \mathrm{~N}$, with a gain of $1 \mathrm{~N} / \mathrm{spike}$, which is the lowest gain that make the $10 \mathrm{~N}$ force production possible. Allowing a context-dependent gain of $0.1 \mathrm{~N} / \mathrm{spike}(\mathrm{A})$, the resolution can be $0.05 \mathrm{~N}$ for the $1 \mathrm{~N}$ force while leaving the solution for the $10 \mathrm{~N}$ force unaffected. $\boldsymbol{b}$, Muscle force plotted as a function of synaptic input and gain according to our model. Depending on the gain, a fixed variance in synaptic inputs translates to different levels of variance in muscle force (variances shown as Gaussian distributions).

tively suppress or enhance the efficacy of 5-HT (see Materials and Methods). This allows determining the serotonergic influence on gain control.

For each trial, subjects ramp up the power force and stabilize it for a fixed duration and subsequently produce a precision force with the left index finger (Fig. 4b). The power force is then switched off while the precision force remains until the end of the trial. How the previous power force modulates the variance of the precision force is the focus of our analyses. We expected that the effects of the intense activation of the descending serotonergic system would gradually decline after the end of the power force for two reasons: (1) the activity of the descending system itself would likely decline relatively slowly; and (2) there is a slow decay of the persistent inward current in motoneurons (Hounsgaard et al., 1988; Heckman and Enoka, 2012). We focus on the precision force variation after the power force is released rather than when the two forces are held concurrently to minimize the effect of divided attention. With these experiments, we can analyze how gain changes in the spinal cord induced by the power force affect the production of a precision force (recall from the above equation that a larger gain will lead to a larger variation in force production).

We find that power forces affect noise levels in the concurrent precision task (Experiment 3; Fig. $4 c-e$ ). More importantly, as expected by our gain control hypothesis, this effect is significant after the drop of power forces, and it decays toward baseline within $\sim 2 \mathrm{~s}$. The intensity of the power force has a strong effect on noise levels.

This intensity effect is observed no matter which effector is used for power force production: the contralateral finger, hand, or the legs. The palm appears to produce the strongest effect. When comparing the $\mathrm{CV}$ during the first second for different effectors in the high force condition, we found a significant difference between the finger and the palm $(p<0.05)$. This might be expected if assuming an approximate somatotopy in the raphe-spinal projections in which the arm muscles (primarily involved for the palm as the effector) has more projections. Thus, we use the palm to elicit gain changes for all our subsequent analyses and additional experiments (Experiments 4 and 5).

If mechanical coupling was underlying the differential effect of the power force on the precision force variance, we would expect that the cross-correlation between two forces should be significantly different from zero and scaled by the power force level. This is, in general, not the case (Fig. $4 f)$. We calculated the cross-correlation between two forces when they are simultaneously on, $0.5 \mathrm{~s}$ before the completion of the power force. It is indistinguishable among three force levels, suggesting that the dependency of variance on power force levels is unlikely to be caused by mechanical coupling.

We performed three control experiments to rule out other possible confounding factors. The involved transients may affect the cognitive burden. In Control Experiment A, we thus reversed the order of two forces, and the systematic effects of the power force onto the precision force are essentially unchanged (Fig. $4 g$, at approximately time 0 and onward). The critical comparison is for the time after the power force is dropped. In addition, we observed that the variance of the precision force does not differ when subjects are expecting different levels of power forces (at $-7 \mathrm{~s}$ ). This suggests there is no priming effect from upcoming power forces.

Second, the precision force is always performed by the left, nondominant hand, and thus handedness might affect the variance results. In Control Experiment B, the hands of the two forces are switched so that the dominant hand performed the precision force and the nondominant hand performed the power force. The noise level in the precision force still follows a similar pattern as in Experiment 3 (Fig. 4h).

Third, primary results are about variance changes in the precision force after the power force exits; what is the natural fluctuation in variance with the precision force only? In Control Experiment C, the same set of subjects from Control Experiment $\mathrm{B}$ was tested to produce the precision force only. The resulting noise level is indistinguishable from that in the low force condition in the dual-force paradigm (Fig. $4 h$ ), suggesting that natural fluctuations in the precision force cannot account for the enhanced noise level associated with power forces. The similarity between the dual-force task (Control Experiment B) and the single-force task (Control Experiment C) also suggests that our findings are not a result of divided attention.

\section{Pharmacological evidence from across-effector tasks}

To establish a role for 5-HT in the observed cross-limb interactions, we directly and selectively manipulated 5-HT activity with drugs and examined performance in the same dual force production task as in Experiment 3. After oral intake of cyproheptadine (Experiment 4), a selective 5-HT receptor antagonist, the excitatory effect of 5-HT is reduced (Barbeau and Rossignol, 1990; Wainberg et al., 1990; Murray et al., 2010), and the precision force is expected to exhibit less variance (Fig. $5 a$ ). This variance reduction should also be more pronounced when the magnitude of the power force, and thus 5-HT levels, increase (Fig. 5b).

We found that subjects show improved precision after cyproheptadine intake and thus performed better after the drop; the opposite would be expected from potential side effects, such as drowsiness, of the drug. In agreement with our hypothesis, there was a greater improvement after larger power forces. After oral intake of paroxetine (Experiment 5), which is a selective 5-HT reuptake inhibitor capable of enhancing the excitatory effect of 5 -HT, the opposite effect is observed (Fig. $5 c, d$ ): the variance in 


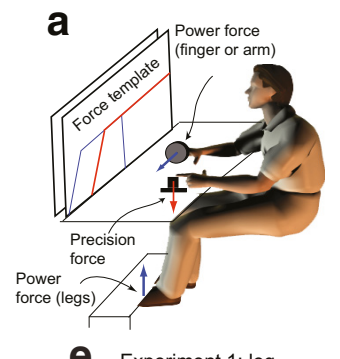

b

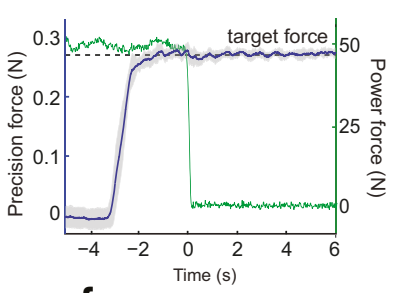

f

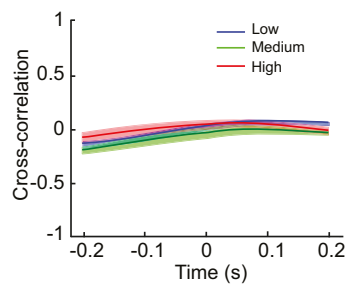

C Experiment 1: palm

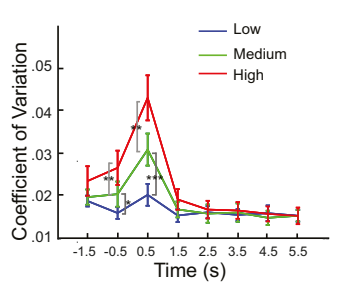

g Control Experiment 1

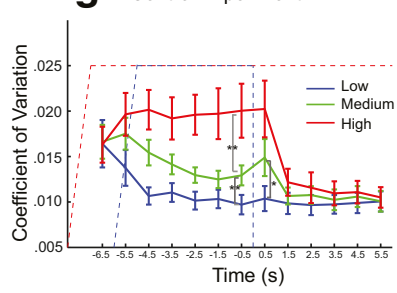

d Experiment 1: finger

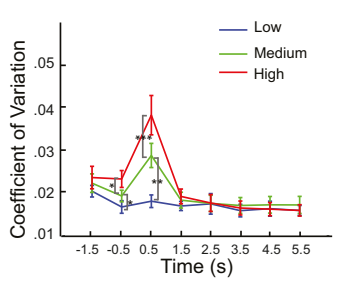

h control Experiment 2 \& 3

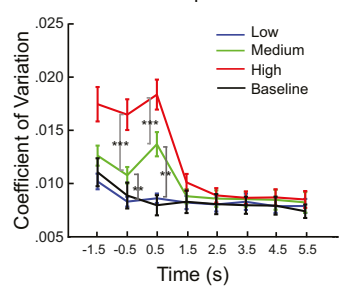

Figure 4. Results from Experiment 3 and Control Experiments A-C. $a$, Experimental setup for Experiments $3-5$ and the control experiments. The lines on the screen represent the force instructions for the precision force (red) and the power force (blue). $\boldsymbol{b}$, Force signals from a typical subject during the medium power force task. Subjects were instructed to produce a precision force with their left index finger (mean shown in blue) while first holding and afterward removing the power force. The green trace depicts a typical power force recording, dropping to half of its amplitude at time 0 . Gray shading denotes SDs across trials. $\boldsymbol{c}-\boldsymbol{e}$, Variance (CV) of the precision force during each second before and after switching off the power force produced by the palm (c), the finger $(\boldsymbol{d})$, and the leg (e). The moment when the power force drops to its half amplitude is defined as time $0.0 \mathrm{n}$ the time axis, $0.5 \mathrm{~s}$ means that the variance is calculated over the first second after time 0 (between 0 and 1 s). Significant differences have been found between power force intensity levels within the $-1,1$, and $2 s$ (not marked in graph) for all effectors. $f$, Cross-correlation of the power force and the precision force is plotted as a function of time lag for three force levels separately. $\boldsymbol{g}$, Results from Control Experiment A, presented in the same format as in $\mathbf{c}$. The dashed lines represent the force instructions for the precision force (red) and the power force (blue), which are now in the reverse order as $\boldsymbol{a}$. Significant differences have been found between power force intensity levels within the $-1,1$, and $2 s$ (not marked in graph). There is no difference found within $-7 \mathrm{~s} . \boldsymbol{h}$, Results from Control Experiments $B$ and $C$, presented in the same format as in $\boldsymbol{c}$. Significant differences have been found between power force intensity levels within the $-1,1$, and $2 s$ (not marked in graph). ${ }^{*} p<0.05,{ }^{* *} p<0.01,{ }^{* * *} p<0.001$.

precision force production increases in all conditions, and this increase is significantly larger with larger power force.

We also found that, throughout the entire period, subjects had lower variance on cyproheptadine and higher variance on paroxetine. Two-way repeated-measures ANOVAs (2 drug conditions $\times 3$ force levels), conducted for each experiment separately, reveal significant main effects of the drugs at different times during a trial (separate tests for the $-1,1,2$, and $6 \mathrm{~s}$; seven of eight tests are significant with $p<0.05$ or $p<0.005$, and the last test comes out marginally significant with $p=0.071$ ). These results indicate that the reduction and increase in variance induced by drugs are significant during the whole trial, before and after the power force is dropped. Therefore, 5-HT also seems to have a reliable baseline influence on resulting variance.

To rule out the possibility of central or peripheral fatigue, we compared the maximum voluntary force before, in the middle of, and after data collection in these dual-task experiments. In Experiment 3, MVCs of all effectors do not change significantly (one-way ANOVA on timing, $p=0.588,0.919,0.723$, and 0.868 for the right finger, palm, leg, and left finger, respectively). For the two drug experiments (Experiments 4 and 5), we performed a 2 (drug) $\times 3$ (timing) repeated-measures ANOVA on MVCs of the finger and the palm. Neither the main effects nor the interaction is significant, indicating that fatigue does not contribute to the observed effects and that MVCs are not affected by cyproheptadine or paroxetine intake.

\section{Discussion}

Here, we have proposed that gain control in the spinal cord is computationally desirable for producing a wide range of muscular forces and established behavioral and pharmacological paradigms to examine its effects and causes. Using reflex assessments, we provided strong evidence that the gain effect of $5-\mathrm{HT}$ is based on spinal mechanisms. These results do not rule out a contribution to gain control from the cortex or other components of the movement system but do provide strong evidence that spinal motoneurons are strongly involved. We have also shown that this gain mechanism is present during force production and can manifest itself as across-effector interactions over time.

Gain control can refer to any phenomenon in which the input-output relationship gets modulated, and hence there are diverse uses of the concept in movement science. In many closedloop situations (e.g., reflexes or perception-action loop), gain is used to refer to the effect of feedback on future movement or forces (Robinson, 1976; Evinger and Manning, 1988; Prochazka, 1989; Brooke et al., 1997; Kawato, 1999; Peterka, 2002). In some sense, any increasing force can be viewed as a result of changing gain of the muscle as increasingly more motor units get recruited (Henneman and Mendell, 1981; Jones et al., 2002). However, in the present study, the gain of force control refers to the slope of input-output function between synaptic inputs (descending drives and afferent signals) and the resulting motor output (force or EMG; Fig. 1a). This is a definition similar to what has been used widely in the perceptual literature (Schwartz and Simoncelli, 2001; Mante et al., 2005): gain is one of two channels relevant to information transmission.

Note that we here specifically refer to the gain control in the spinal cord. In cortical neurons, synaptic noise can markedly decrease gain in response to input (Chance et al., 2002). Spinal motoneurons work in a different synaptic processing regimen, having long spike afterhyperpolarizations and relatively regular spiking outputs (CVs of firing in motoneurons in humans during voluntary contractions rarely exceed 0.2 ; Heckman and Enoka, 2012). Instead, gain control occurs via neuromodulatory inputs, such as 5-HT, that increase persistent inward currents to amplify incoming synaptic current. 
a

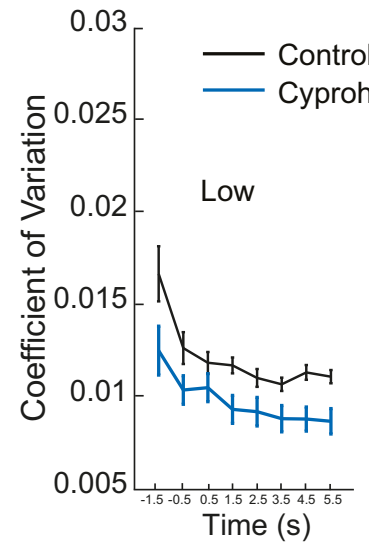

C

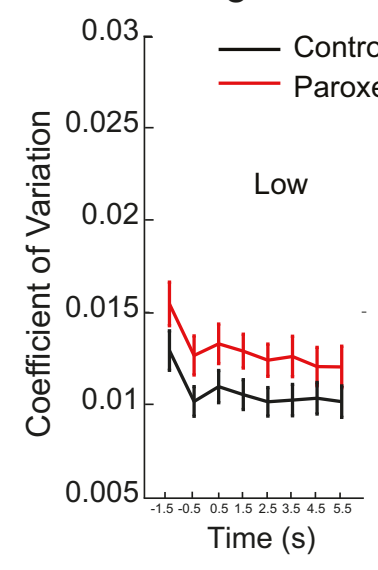

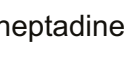

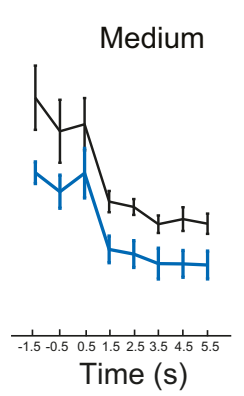

$-1.5-0.5 \quad 0.51 .52 .53 .5$
Time $(\mathbf{s})$ b
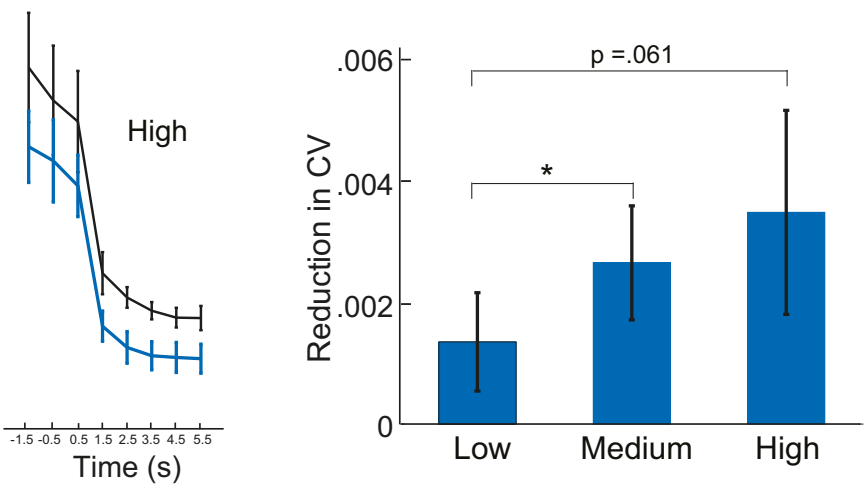

d

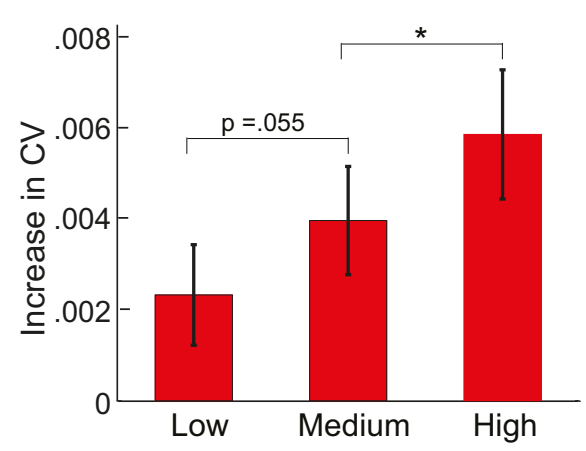

Figure 5. Results from Experiments 4 and 5. a, Variance in the precision force plotted as a function of time, with or without cyproheptadine intake. The data from different power force conditions are plotted separately. $\boldsymbol{b}$, Variance reduction (within the 1st second) induced by cyproheptadine intake is plotted as a function of the power force. $\boldsymbol{c}$, Variance in the precision force as a function of time, with or without paroxetine intake. $\boldsymbol{d}$, Variance increase (within the 1st second) induced by paroxetine intake is plotted as a function of the power force. Error bars denote mean \pm SEM across $n=8$ subjects. ${ }^{*} p<0.05$.

Previous studies provide significant neurophysiological evidence supporting a role for 5-HT in the excitability of motor neurons (Heckman et al., 2003; Hultborn et al., 2004). The behavior of the brainstem-spinal neuromodulatory system is state dependent: quiescent in the sleeping state and tonically active in the waking state (Jacobs et al., 2002). In addition, it appears likely that the 5-HT projection to the cord increases its activity with increasing motor output (Jacobs and Fornal, 1993; Jacobs et al., 2002). Thus, the brainstem-spinal cord neuromodulatory system could adaptively match the gain of motoneurons to the demands of both precise and intense motor behaviors. This kind of gain control is particularly useful in cases in which communication channels are narrow. The descending pyramidal tract, for instance, contains $<10^{6}$ axons (Kuypers, 1981), which is relatively narrow given that there are both a large number of cortical motor neurons and a large number of involved muscles and fibers. Adaptive gain control can support generating large motor output without sacrificing precision for small motor output.

It might be asked whether the introduction of a gain would also introduce noise, which might undo the advantage of using gain control. However, if the gain changes slowly, then the transmission of the gain can have a large time constant. Because SEs of firing rate estimates scale linearly with the inverse square root of integration time, long integration time constants translate into small variance for slowly changing gains. Thus, the effect of noise in gain is negligible and it is ignored in our simple model (see equation in Results).

Although the neuromodulatory gain control is advantageous in terms of offering precise control over a large range with limited bandwidth, the obvious disadvantage for this strategy is the unnecessary coupling between effectors. This unspecific control can be negated by reciprocal inhibition mediated by spindle Ia afferents and Ia interneurons (Hyngstrom et al., 2007; Bui et al., 2008). Dendritic persistent inward current, which is the main mechanism of serotonergic control of motoneuron gain, is highly sensitive to this inhibition (Hultborn et al., 2003; Kuo et al., 2003). Thus, the nervous system can still offer specific control with a diffusive neuromodulation background if the descending motor commands are coupled to both brainstem monoaminergic nuclei and to reciprocal inhibitory interneurons in the spinal cord (Heckman et al., 2008).

Our findings from spinal reflexes provided direct support for a spinal locus for the 5-HT effect. In Experiment 1, we demonstrated that the response of the tendon vibration reflex can be amplified by selectively manipulating 5 -HT efficacy. We consider a descending contribution unlikely to account for the gain effect we observed in Experiment 1 for two reasons. First, the subject was instructed to relax during the whole duration of a trial. The vibration was applied at a random time in each trial to minimize anticipation. These measures prevented voluntary intervention 
of reflexive responses. In fact, the difference in reflexive response between 5 -HT drug conditions is evidenced immediately after application of vibration (Fig. 1b), suggesting that the gain effect is not mediated by feedback.

Second, previous studies on tendon vibration reflex in animals and humans established that this type of reflex is essentially monosynaptic. In animal preparations, the tendon vibration reflex is well established as being attributable to the monosynaptic connections of Ia afferents onto motoneurons (Matthews, 1972). In humans, vibration must be applied via the skin and thus activates additional afferents (Fallon and Macefield, 2007), but the overall form of the reflex is identical to that in the animal. The tendon vibration reflexes studied here behaved just as in animal preparations, including the tendency for a slow increase and then decrease that is a hallmark of the short-term plastic behavior exhibited by the voltage-sensitive Ca channels that generate persistent inward currents (Powers and Binder, 2001).

However, an unconscious contribution in human subjects, although unlikely, is still possible. This possibility was one reason that prompted us to conduct Experiment 2 with tendon tap reflex, which is much too brief to be affected by changes in descending drives. We applied tendon taps to the knee instead of the wrist because of the complexity of the wrist tendon structure. Experiment 2 further demonstrates the spinal loci of gain control of motor output; both the magnitude and the gain of the shortlatency $(<50 \mathrm{~ms})$ tendon reflex is either amplified or suppressed with differing 5-HT medications in individuals with reduced cortical drive.

Although the effect of 5-HT that we discovered in Experiments $3-5$ could in principle also be outside of the spinal cord, previous physiological work (Fetz et al., 2000; Powers and Binder, 2001; Hultborn et al., 2004; Heckman et al., 2008; Dayan and Huys, 2009) makes it very likely that the action is in the spinal cord. For example, our administration of drugs might affect the cortical control of force. However, for a single dose, their effects are most potent on the spinal cord, because previous studies revealed that a moderate to high dose of 5-HT in an animal preparation can increase motoneuron throughput gain by as much as fivefold (Lee and Heckman, 2000; Hultborn et al., 2003). In addition, motoneurons are densely covered in synaptic boutons containing 5-HT, which provide direct, monosynaptic connections from the brainstem (Alvarez et al., 1998; Montague et al., 2013). In fact, the number of 5-HT synapses is larger than the number of synapses from muscle spindle Ia afferents mediating the tendon tap and vibration reflexes (Alvarez et al., 1998). Hence, although we cannot exclude cortical effect for cross-limb interaction, previous findings and our results on spinal reflexes support the role of 5-HT on the spinal level.

A possible cortical mechanism is that the variance increase in our psychophysical experiments results from divided attention such that a higher power force is associated with a greater attentional effect. However, there are two results arguing against this interpretation. First, we observe differing variance with pharmaceutical interventions long after the power force is stopped. This cannot be explained by attention because there is no more dual task. Second, tendon reflexes, which are spinally mediated, were clearly modified by the changes in 5-HT levels in the direction consistent with the gain changes that we postulate altered force variances. Thus, these pieces of evidence suggest that divided attention cannot explain the observed variance increase in force production.

It is noteworthy that our experiments involving pharmaceutical intervention of 5-HT efficacy did not change the MVC. Dur- ing the MVC, muscle fibers are maximally recruited, and thus the force output will not increase further even when the 5-HT efficacy is amplified. Conversely, the MVC is still unaffected when the 5-HT efficacy is reduced by a selective 5-HT reuptake inhibitor. We postulate in this case that other neuromodulators help in amplifying the gain when large force production is demanded, as revealed by single-cell studies (Miles et al., 2007; Power et al., 2010). Thus, this result is consistent with the notion that other neuromodulators might also play a role in gain control. For instance, animal studies typically found that both 5-HT and NE have an amplification effect. Here we focus on 5-HT because NE is closely related to arousal, making 5-HT a more likely candidate for generic neuromodulatory gain control. Moreover, the 5-HT projection to the spinal cord increases its activity with increasing motor output (Jacobs et al., 2002). Finally, 5-HT alone can produce an equally strong amplification effect as both 5-HT and NE combined, as shown in an animal preparation study (Harvey et al., 2006).

There are many previously described changes in excitability of the human motor system. An intensive volitional contraction will result in reflex potentiation in both the contracting (Enoka et al., 1980; Gregory et al., 1990) and remote (Delwaide and Toulouse, 1981; Dowman and Wolpaw, 1988; Miyahara et al., 1996; Zehr and Stein, 1999; Stam, 2000) muscles, as well as an enhancement of corticospinal tract excitability (Kawakita et al., 1991; Péréon et al., 1995; Stedman et al., 1998; Boroojerdi et al., 2000; Tazoe et al., 2009). The proposed mechanisms underlying this remote alteration in the excitability of the motor system range from decreased presynaptic inhibition of Ia terminals to widespread increases in cortical excitability.

We cannot completely exclude potential contributions from these mechanisms; however, the systematic patterns elicited by pharmacologically manipulating the efficacy of 5-HT on both volitional and reflexive pathways provide firm support for both the neuromodulatory and spinal origins of our findings. Moreover, our current findings are fully consistent with these previous investigations, and the widespread actions of 5-HT on the gain control of spinal neurons may be an unaccounted for mechanism that would aid in unifying these previous interpretations. It is now clear that future studies on remote effects should take neuromodulatory gain control in the spinal cord into consideration.

Targeting the serotonergic system has been shown recently to be a promising strategy to improve function and reduce spasticity after spinal cord injury (Wainberg et al., 1990; Murray et al., 2010). In terms of behavior, it is important to realize that, when multiple effectors are involved in movement, their noise levels are not independent from one another, because gain control makes them dependent. Gain control in the spinal cord promises to be important topic for research in rehabilitation and motor control.

\section{References}

Alvarez FJ, Pearson JC, Harrington D, Dewey D, Torbeck L, Fyffe RE (1998) Distribution of 5-hydroxytryptamine-immunoreactive boutons on alpha-motoneurons in the lumbar spinal cord of adult cats. J Comp Neurol 393:69-83. CrossRef Medline

Aston-Jones G, Chen S, Zhu Y, Oshinsky ML (2001) A neural circuit for circadian regulation of arousal. Nat Neurosci 4:732-738. CrossRef Medline

Barbeau H, Rossignol S (1990) The effects of serotonergic drugs on the locomotor pattern and on cutaneous reflexes of the adult chronic spinal cat. Brain Res 514:55-67. CrossRef Medline

Boroojerdi B, Battaglia F, Muellbacher W, Cohen LG (2000) Voluntary teeth clenching facilitates human motor system excitability. Clin Neurophysiol 111:988-993. CrossRef Medline

Bowker RM, Westlund KN, Sullivan MC, Coulter JD (1982) Organization 
of descending serotonergic projections to the spinal cord. In: Descending pathways to the spinal cord. Progress in Brain Res (Kuypers HGJM, Martin GF, eds), pp 239-265. Amsterdam: Elsevier.

Brooke JD, Cheng J, Collins DF, Mcllroy WE, Misiaszek JE, Staines WR (1997) Sensori-sensory afferent conditioning with leg movement: gain control in spinal reflex and ascending paths. Prog Neurobiol 51:393-421. CrossRef Medline

Bui TV, Grande G, Rose PK (2008) Multiple modes of amplification of synaptic inhibition to motoneurons by persistent inward currents. J Neurophysiol 99:571-582. CrossRef Medline

Carlton L, Newell K (1993) Force variability and characteristics of force production. In: Force variability, pp 15-36. Champaign, IL: Human Kinetics.

Carp JS, Rymer WZ (1986) Enhancement by serotonin of tonic vibration and stretch reflexes in the decerebrate cat. Exp Brain Res 62:111-122. Medline

Chance FS, Abbott LF, Reyes AD (2002) Gain modulation from background synaptic input. Neuron 35:773-782. CrossRef Medline

Churchland MM, Yu BM, Cunningham JP, Sugrue LP, Cohen MR, Corrado GS, Newsome WT, Clark AM, Hosseini P, Scott BB, Bradley DC, Smith MA, Kohn A, Movshon JA, Armstrong KM, Moore T, Chang SW, Snyder LH, Lisberger SG, Priebe NJ, et al. (2010) Stimulus onset quenches neural variability: a widespread cortical phenomenon. Nat Neurosci 13:369378. CrossRef Medline

Cushing S, Bui T, Rose PK (2005) Effect of nonlinear summation of synaptic currents on the input-output properties of spinal motoneurons. J Neurophysiol 94:3465-3478. CrossRef Medline

Dayan P, Huys QJM (2009) Serotonin in affective control. Annu Rev Neurosci 32:95-126. CrossRef Medline

Delwaide PJ, Toulouse P (1981) Facilitation of monosynaptic reflexes by voluntary contraction of muscle in remote parts of the body. Mechanisms involved in the Jendrassik Manoeuvre. Brain 104:701-709. CrossRef Medline

Devanne H, Lavoie BA, Capaday C (1997) Input-output properties and gain changes in the human corticospinal pathway. Exp Brain Res 114:329-338. CrossRef Medline

Dowman R, Wolpaw JR (1988) Jendrassik maneuver facilitates soleus $\mathrm{H}$-reflex without change in average soleus motoneuron pool membrane potential. Exp Neurol 101:288-302. CrossRef Medline

Enoka RM, Hutton RS, Eldred E (1980) Changes in excitability of tendon tap and Hoffmann reflexes following voluntary contractions. Electroencephalogr Clin Neurophysiol 48:664-672. CrossRef Medline

Evinger C, Manning KA (1988) A model system for motor learning: adaptive gain control of the blink reflex. Exp Brain Res 70:527-538. Medline

Fallon JB, Macefield VG (2007) Vibration sensitivity of human muscle spindles and Golgi tendon organs. Muscle Nerve 36:21-29. CrossRef Medline

Fetz EE, Perlmutter SI, Prut Y (2000) Functions of mammalian spinal interneurons during movement. Curr Opin Neurobiol 10:699-707. CrossRef Medline

Fullerton GS, Cattell JM (1892) On the perception of small differences. University of Pennsylvania Philosophical Series 2. Philadelphia: University of Pennsylvania.

Gregory JE, Mark RF, Morgan DL, Patak A, Polus B, Proske U (1990) Effects of muscle history on the stretch reflex in cat and man. J Physiol 424:93107. Medline

Harvey PJ, Li X, Li Y, Bennett DJ (2006) 5-HT2 receptor activation facilitates a persistent sodium current and repetitive firing in spinal motoneurons of rats with and without chronic spinal cord injury. J Neurophysiol 96:1158-1170. CrossRef Medline

Heckman C, Enoka RM (2012) Motor unit. Comp Physiol 2:2629-2686. CrossRef

Heckman CJ, Lee RH, Brownstone RM (2003) Hyperexcitable dendrites in motoneurons and their neuromodulatory control during motor behavior. Trends Neurosci 26:688-695. CrossRef Medline

Heckman CJ, Hyngstrom AS, Johnson MD (2008) Active properties of motoneurone dendrites: diffuse descending neuromodulation, focused local inhibition. J Physiol 586:1225-1231. CrossRef Medline

Henneman E, Mendell L (1981) Functional organization of motoneuron pool and its inputs. Handbook of physiology: the nervous system motor control, pp 423-507. Bethesda, MD: American Physiological Society.

Holstege JC, Kuypers HG (1987) Brainstem projections to spinal motoneurons: an update. Neuroscience 23:809-821. CrossRef Medline
Hounsgaard J, Hultborn H, Jespersen B, Kiehn O (1988) Bistability of alpha-motoneurones in the decerebrate cat and in the acute spinal cat after intravenous 5-hydroxytryptophan. J Physiol 405:345-367. Medline

Hultborn H, Denton ME, Wienecke J, Nielsen JB (2003) Variable amplification of synaptic input to cat spinal motoneurones by dendritic persistent inward current. J Physiol 552:945-952. CrossRef Medline

Hultborn H, Brownstone RB, Toth TI, Gossard JP (2004) Key mechanisms for setting the input-output gain across the motoneuron pool. Prog Brain Res 143:77-95. Medline

Hyngstrom AS, Johnson MD, Miller JF, Heckman CJ (2007) Intrinsic electrical properties of spinal motoneurons vary with joint angle. Nat Neurosci 10:363-369. CrossRef Medline

Jacobs BL, Fornal CA (1993) 5-HT and motor control: a hypothesis. Trends Neurosci 16:346-352. CrossRef Medline

Jacobs BL, Martín-Cora FJ, Fornal CA (2002) Activity of medullary serotonergic neurons in freely moving animals. Brain Res Brain Res Rev 40:45-52. CrossRef Medline

Jones KE, Hamilton AFC, Wolpert DM (2002) Sources of signal-dependent noise during isometric force production. J Neurophysiol 88:1533-1544. Medline

Kawakita H, Kameyama O, Ogawa R, Hayes KC, Wolfe DL, Allatt RD (1991) Reinforcement of motor evoked potentials by remote muscle contraction. J Electromyogr Kinesiol 1:96-106. CrossRef Medline

Kawato M (1999) Internal models for motor control and trajectory planning. Curr Opin Neurobiol 9:718-727. CrossRef Medline

Kernell D (2006) The motoneurone and its muscle fibres. Oxford: Oxford UP.

Kuo JJ, Lee RH, Johnson MD, Heckman HM, Heckman CJ (2003) Active dendritic integration of inhibitory synaptic inputs in vivo. J Neurophysiol 90:3617-3624. CrossRef Medline

Kuypers HG (1981) Anatomy of the descending pathways. In: The nervous system, Handbook of physiology (Brooks V, ed). Baltimore: Williams and Wilkins.

Lee RH, Heckman CJ (2000) Adjustable amplification of synaptic input in the dendrites of spinal motoneurons in vivo. J Neurosci 20:6734-6740. Medline

Mante V, Frazor RA, Bonin V, Geisler WS, Carandini M (2005) Independence of luminance and contrast in natural scenes and in the early visual system. Nat Neurosci 8:1690-1697. CrossRef Medline

Marino RJ, Graves DE (2004) Metric properties of the ASIA motor score: subscales improve correlation with functional activities. Arch Phys Med Rehabil 85:1804-1810. CrossRef Medline

Matthews PB (1972) Mammalian muscle receptors and their central actions. London: Arnold.

Miles GB, Hartley R, Todd AJ, Brownstone RM (2007) Spinal cholinergic interneurons regulate the excitability of motoneurons during locomotion. Proc Natl Acad Sci U S A 104:2448-2453. CrossRef Medline

Miyahara T, Hagiya N, Ohyama T, Nakamura Y (1996) Modulation of human soleus $\mathrm{H}$ reflex in association with voluntary clenching of the teeth. J Neurophysiol 76:2033-2041. Medline

Montague SJ, Fenrich KK, Mayer-Macaulay C, Maratta R, Neuber-Hess MS, Rose PK (2013) Nonuniform distribution of contacts from noradrenergic and serotonergic boutons on the dendrites of cat splenius motoneurons. J Comp Neurol 521:638-656. CrossRef Medline

Murray KC, Nakae A, Stephens MJ, Rank M, D’Amico J, Harvey PJ, Li X, Harris RLW, Ballou EW, Anelli R, Heckman CJ, Mashimo T, Vavrek R, Sanelli L, Gorassini MA, Bennett DJ, Fouad K (2010) Recovery of motoneuron and locomotor function after spinal cord injury depends on constitutive activity in 5-HT2C receptors. Nat Med 16:694-700. CrossRef Medline

Naito E, Roland PE, Ehrsson HH (2002) I feel my hand moving: a new role of the primary motor cortex in somatic perception of limb movement. Neuron 36:979-988. CrossRef Medline

Newell KM, Carlton LG (1988) Force variability in isometric responses. J Exp Psychol Hum Percept Perform 14:37-44. CrossRef Medline

Péréon Y, Genet R, Guihéneuc P (1995) Facilitation of motor evoked potentials: timing of Jendrassik maneuver effects. Muscle Nerve 18:1427-1432. CrossRef Medline

Peterka RJ (2002) Sensorimotor integration in human postural control. J Neurophysiol 88:1097-1118. Medline

Power KE, McCrea DA, Fedirchuk B (2010) Intraspinally mediated statedependent enhancement of motoneurone excitability during fictive 
scratch in the adult decerebrate cat. J Physiol 588:2839-2857. CrossRef Medline

Powers RK, Binder MD (2001) Input-output functions of mammalian motoneurons. Rev Physiol Biochem Pharmacol 143:137-263. CrossRef Medline

Prochazka A (1989) Sensorimotor gain control: a basic strategy of motor systems? Prog Neurobiol 33:281-307. CrossRef Medline

Robinson DA (1976) Adaptive gain control of vestibuloocular reflex by the cerebellum. J Neurophysiol 39:954-969. Medline

Schmidt RA, Zelaznik H, Hawkins B, Frank JS, Quinn Jr JT (1979) Motoroutput variability: a theory for the accuracy of rapid motor acts. Psychol Rev 86:415. CrossRef Medline

Schwartz O, Simoncelli EP (2001) Natural signal statistics and sensory gain control. Nat Neurosci 4:819-825. CrossRef Medline

Smirnakis SM, Berry MJ, Warland DK, Bialek W, Meister M (1997) Adaptation of retinal processing to image contrast and spatial scale. Nature 386:69-73. CrossRef Medline

Stam J (2000) Jendrassik's maneuver. Neurological eponyms, p 143. Oxford, UK: Oxford UP.
Stedman A, Davey NJ, Ellaway PH (1998) Facilitation of human first dorsal interosseous muscle responses to transcranial magnetic stimulation during voluntary contraction of the contralateral homonymous muscle. Muscle Nerve 21:1033-1039. CrossRef Medline

Tazoe T, Sakamoto M, Nakajima T, Endoh T, Shiozawa S, Komiyama T (2009) Remote facilitation of supraspinal motor excitability depends on the level of effort. Eur J Neurosci 30:1297-1305. CrossRef Medline

Teich MC, Heneghan C, Lowen SB, Ozaki T, Kaplan E (1997) Fractal character of the neural spike train in the visual system of the cat. J Opt Soc Am A Opt Image Sci Vis 14:529-546. CrossRef Medline

Wainberg M, Barbeau H, Gauthier S (1990) The effects of cyproheptadine on locomotion and on spasticity in patients with spinal cord injuries. J Neurol Neurosurg Psychiatry 53:754-763. CrossRef Medline

Zehr EP, Stein RB (1999) Interaction of the Jendrassik maneuver with segmental presynaptic inhibition. Exp Brain Res 124:474-480. CrossRef Medline

Zhao HB, Santos-Sacchi J (1999) Auditory collusion and a coupled couple of outer hair cells. Nature 399:359-362. CrossRef Medline 